\title{
The radial distribution in nearby galaxies of the ionizing field of radiation of HII regions using photoionization models
}

\author{
Enrique Pérez-Montero, Rubén García-Benito and José M. Vílchez \\ Instituto de Astrofísica de Andalucía - CSIC, Apdo 3004, E-18080, Granada, Spain
}

\begin{abstract}
HiI regions in galaxy disks can be used as a powerful tool to trace the radial distribution of several of their properties and shed some light on the different relevant processes on galaxy formation and evolution. Among the properties that can be extracted from the study of the ionized gas are the metallicity, the excitation and the hardness of the ionizing field of radiation. In this contribution we focus on the determination of both the ionization parameter $(U)$ and the effective temperature of the ionizing clusters $\left(T_{*}\right)$ by means of a bayesian-like comparison between the observed relative fluxes of several emission-lines with the predictions from a set of photoionization models. We also show the implications that the use of our method has for the study of the radial variation of both $U$ and $T_{*}$ in some very well-studied disk galaxies of the Local Universe.
\end{abstract}

Keywords. methods: data analysis - ISM: abundances - galaxies: abundances

The equivalent effective temperature $\left(T_{*}\right)$ of the ionizing field of radiation is one of the functional parameters that dominates the relative flux of the most prominent emission lines in HII regions. In unresolved objects Vilchez \& Pagel (1988) propose the so-called softness parameter, defined as $\log \eta^{\prime}=\log ([\mathrm{OII}] 3727 \AA /[\mathrm{OIII}] 4959,5007$ $\AA) /([\mathrm{SII}] 6717,31 \AA /[\mathrm{SIII}] 9069,9532 \AA)$ in the optical range, that can be an estimator for $T_{*}$. According to Pérez-Montero \& Vílchez (2009) most nearby disk galaxies present negative radial slopes of $\log \eta \prime$ what could be interpreted as a radial hardening of the ionizing stellar radiation in these objects. To confirm this trend we built a large grid of photoionization models covering a wide range in $T_{*}$, metallicity, and ionization parameter and we designed a code, called HII-CHI-MISTRY-TEFF with similar features as those described in Pérez-Montero (2014), which finds by means a bayesian-like approach, the best solution to fit the relative observed intensities of the four emission lines defined in $\log \eta \prime$. We applied our code to a large sample of HII regions in the galaxies of the CHAOS project, described in Berg et al. (2015), with good-quality spectroscopical optical data of the four required emission lines and a determination of the metallicity based on the direct method. We verified that the analyzed galaxies present radial increases of $T_{*}$ that correlate with the negative slopes of their metallicity gradients. On the contrary, no radial variations of the ionization parameter are found for the same objects.

\section{References}

Berg, D. A., Skillman, E. D., Croxall, K. V., et al. 2015, ApJ, 806, 16

Pérez-Montero, E. 2014, MNRAS, 441, 2663

Pérez-Montero, E., \& Vílchez, J. M. 2009, MNRAS, 400, 1721

Vilchez, J. M., \& Pagel, B. E. J. 1988, MNRAS, 231, 257 